 \\ MOUNTaiNs
}

Mathematical Publications

\section{BOUNDEDNESS AND OSCILLATION OF THIRD ORDER NEUTRAL DIFFERENTIAL EQUATIONS}

\author{
Božena Mihalíková - Eva Kostiková
}

ABSTRACT. The relationship between boundedness and oscillation of solutions of the third order neutral differential equations are presented.

\section{Introduction}

In this paper we consider third order neutral differential equations of the form

$$
\left(r_{2}(t)\left(r_{1}(t)\left(x(t)-p x(\tau(t))^{\prime}\right)^{\prime}\right)^{\prime}+q(t) f(x(\sigma(t)))\right), \quad t \geq t_{0}
$$

and the following conditions are assumed to hold:

$\left(\mathrm{H}_{1}\right) 0<p<1$;

$\left(\mathrm{H}_{2}\right) \tau \in C\left(\left[t_{0}, \infty\right), R\right), \tau(t)<t, \tau$ is strictly increasing, $\lim _{t \rightarrow \infty} \tau(t)=\infty$ and we define

$$
\tau^{0}(t)=t, \quad \tau^{i}(t)=\tau\left(\tau^{i-1}(t)\right), \quad i=1,2, \ldots ;
$$

$\left(\mathrm{H}_{3}\right) q, r_{j} \in C\left(\left[t_{0}, \infty\right),(0, \infty)\right)$, and

$$
\int_{0}^{\infty} r_{j}(t) \mathrm{d} t=\infty, \quad j=1,2
$$

$\left(\mathrm{H}_{4}\right) \sigma \in C^{1}\left(\left[t_{0}, \infty\right), R\right), \sigma^{\prime}(t)>0, \sigma(t)<t$ and $\lim _{t \rightarrow \infty} \sigma(t)=\infty$;

$\left(\mathrm{H}_{5}\right) f \in C(R, R), f$ is nondecreasing, $u f(u)>0$ for $u \neq 0$ and

$$
\liminf _{u \rightarrow 0} \frac{f(u)}{u}>0 \text {. }
$$

2000 Mathematics Subject Classification: $34 \mathrm{~K} 15,34 \mathrm{~K} 10$.

Keywords: neutral equation, oscillatory solution. 


\section{BOŽENA MIHALÍKOVÁ - EVA KOSTIKOVÁ}

For the sake of convenience we introduce the following notation:

$$
\begin{aligned}
z(t) & =x(t)-p x(\tau(t)), \\
L_{0} z(t) & =z(t), \\
L_{i} z(t) & =r_{i}(t) \frac{\mathrm{d}}{\mathrm{d} t} L_{i-1} z(t), \quad i=1,2, \\
L_{3} z(t) & =\frac{\mathrm{d}}{\mathrm{d} t} L_{2} z(t) .
\end{aligned}
$$

Asymptotic properties of solutions of differential equations (ordinary, with deviating argument) of the third order have been subject of intensive studying in the literature. This problem for neutral differential equations has received considerable attention in recent years (see the references cited, for example, in [6]. Boundedness and oscillation are, generally speaking, independent properties. Nevertheless, there exists a precise relation between them. In this paper we give sufficient conditions for the bounded solution of the equation (11) to be oscillatory.

By a solution of the equation (1) we mean a continuous function $x(t)$ satisfying (11) on $\left[t_{x}, \infty\right)$ such that $L_{i} z(t), 0 \leq i \leq 3$ exist and are continuous on $\left[t_{x}, \infty\right)$. A nontrivial solution of (11) is called oscillatory if it has arbitrarily large zeros; otherwise it is called nonoscillatory.

\section{Properties of nonoscillatory solutions}

We begin with analyzing of the asymptotic behavior of possible non-oscillatory solutions of the equation (11). Let $x$ be a non-oscillatory solution of (11) on $\left[t_{0}, \infty\right)$. From the equation (11) it follows that the function $z$ has to be eventually of constant sign, so either

$$
x(t) z(t)>0,
$$

or

$$
x(t) z(t)<0,
$$

for all sufficiently large $t$. Denote by $N^{+}$[or $N^{-}$] the set of all non-oscillatory solutions $x$ of the equation (1) such that (3) [or (4)] is satisfied.

The following lemmas will be useful in the proofs of main results.

Lemma 2.1. Let $\left(\mathrm{H}_{1}\right),\left(\mathrm{H}_{2}\right)$ hold and $x$ be continuous nonoscillatory solution of the functional inequality (3). Then

$$
\lim _{t \rightarrow \infty} x(t)=0, \quad \lim _{t \rightarrow \infty} z(t)=0 .
$$


Lemma 2.2. Let $\left(\mathrm{H}_{1}\right),\left(\mathrm{H}_{2}\right)$ hold and $x$ be continuous nonoscillatory solution of the functional inequality (4). If

$$
\lim _{t \rightarrow \infty} z(t)=0, \text { then } \lim _{t \rightarrow \infty} x(t)=0 .
$$

These lemmas are modifications of the Lemma 2.1 in the paper [7] and the Lemma 2 in the paper [8].

\section{Main results}

Theorem 3.1. Let the conditions $\left(\mathrm{H}_{1}\right)-\left(\mathrm{H}_{5}\right)$ hold. If

$$
\limsup _{t \rightarrow \infty} \int_{\sigma(t)}^{t} q(s) \int_{\sigma(s)}^{\sigma(t)} \frac{1}{r_{1}(u)} \int_{u}^{\sigma(t)} \frac{1}{r_{2}(v)} \mathrm{d} v \mathrm{~d} u \mathrm{~d} s>0,
$$

then every bounded solution $x$ of (11) is either oscillatory or tends to zero as $t \rightarrow \infty$.

Proof. Let $x$ be a bounded non-oscillatory solution of (11) on the interval $\left[t_{0}, \infty\right)$. Without loss of generality we may assume that $x(t)>0, x(\sigma(t))>0$ for $t \geq t_{1} \geq t_{0}$. Then $z$ is bounded and nonoscillatory, too. The equation (11) implies that $L_{2} z$ is a decreasing function and there are two possibilities:

I. $L_{2} z(t)<0, t \geq t_{2} \geq t_{1}$;

II. $L_{2} z(t)>0, t \geq t_{2} \geq t_{1}$.

Let $\mathbf{I}$. hold. Then there exist a $t_{3} \geq t_{2}$ and a constant $A<0$ such that

$$
L_{2} z(t)<A, \quad \text { resp. } \quad \frac{\mathrm{d}}{\mathrm{d} t} L_{1} z(t)<\frac{A}{r_{2}(t)}, \quad \text { for } \quad t \geq t_{3} .
$$

Integrating the last inequality and using $\left(\mathrm{H}_{3}\right)$ we get $L_{1} z(t) \rightarrow-\infty$ as $t \rightarrow \infty$ and then there exists a $t_{4} \geq t_{3}$ and a constant $B<0$ such that

$$
L_{1} z(t) \leq B, \quad \text { resp. } \quad \frac{\mathrm{d}}{\mathrm{d} t} L_{0} z(t)<\frac{B}{r_{1}(t)}, \quad \text { for } \quad t \geq t_{4} .
$$

Again integrating this inequality from $t_{4}$ to $t$ and using $\left(\mathrm{H}_{3}\right)$ we get $z(t) \rightarrow-\infty$ as $t \rightarrow \infty$. This yields that $x \in N^{-}$and this contradicts the Lemma 2.1 .

Now we assume that II. holds, i.e., $L_{2} z(t)>0, t \geq t_{2}$. Then $L_{1} z$ is the increasing function and we have two possibilities for $L_{1} z$ :

(a) $L_{1} z(t)>0$ for $t \geq t_{3} \geq t_{2}$;

(b) $L_{1} z(t)<0$ for $t \geq t_{3} \geq t_{2}$. 


\section{BOŽENA MIHALÍKOVÁ - EVA KOSTIKOVÁ}

In the case (a) there exist a $t_{4} \geq t_{3}$ and a constant $C>0$ such that

$$
L_{1} z(t) \geq C>0, \quad \text { resp. } \quad \frac{\mathrm{d}}{\mathrm{d} t} L_{0} z(t)>\frac{C}{r_{1}(t)}, \quad \text { for } \quad t \geq t_{4} .
$$

The last inequality and $\left(\mathrm{H}_{3}\right)$ imply $\lim _{t \rightarrow \infty} z(t)=\infty$. This means that $z(t)>0$ and according to (2) we get $x(t) \geq z(t)$ for all sufficiently large $t$. Then $\lim _{t \rightarrow \infty} x(t)=\infty$, which contradicts the boundedness of $x$.

Let the case (b) hold. Then $z$ is decreasing and there exists $\lim _{t \rightarrow \infty} z(t)=\alpha$. If $z(t)<0$ for $t \geq t_{4} \geq t_{3}$, then $\alpha<0$, which contradicts the Lemma 2.1. Therefore $z(t)>0$ for $t \geq t_{4} \geq t_{3}$ and $\alpha \geq 0$. We shall prove that $\alpha=0$.

Let $\alpha>0$. Integration of the identity $L_{1} z(t)=L_{1} z(t)$ from $\sigma(s)$ to $\sigma(t)$, $t>s>T \geq t_{4}$ leads to

$$
z(\sigma(t))-z(\sigma(s))=\int_{\sigma(s)}^{\sigma(t)} \frac{1}{r_{1}(u)} L_{1} z(u) \mathrm{d} u .
$$

Integrating the identity $L_{2} z(t)=L_{2} z(t)$ from $u$ to $\sigma(t), T<s<u<t$ we obtain

$$
-L_{1} z(u) \geq L_{1} z(\sigma(t))-L_{1} z(u)=\int_{u}^{\sigma(t)} \frac{1}{r_{2}(v)} L_{2} z(v) \mathrm{d} v,
$$

for $t \geq u \geq T$. We know that $L_{2} z$ is decreasing and so the last inequality implies

$$
L_{1} z(u) \leq-L_{2} z(\sigma(t)) \int_{u}^{\sigma(t)} \frac{1}{r_{2}(v)} \mathrm{d} v
$$

Combining (5), (6) we get

$$
\begin{aligned}
& z(\sigma(t))-z(\sigma(s)) \leq-L_{2} z(\sigma(t)) \int_{\sigma(s)}^{\sigma(t)} \frac{1}{r_{1}(u)} \int_{u}^{\sigma(t)} \frac{1}{r_{2}(v)} \mathrm{d} v \mathrm{~d} u, \quad t>s \geq T, \\
& \text { for } t \geq s \geq T
\end{aligned}
$$

Let us define a function

$$
F(s, t)=\left(L_{2} z(s)-L_{2}(\sigma(t))\right) \int_{s}^{t} \frac{z^{\prime}(\sigma(u)) \sigma^{\prime}(u)}{f(z(\sigma(u)))} \mathrm{d} u, \quad t \geq s \geq T .
$$

Hence $F(t, t)=0=F(\sigma(t), t)$. Deriving $F(s, t)$ partially with respect to $s$, using the equation (11), $\left(\mathrm{H}_{4}\right)$, monotonicity of $z, f$, positivity of $L_{2} z$ we get

$$
F_{s}^{\prime}(s, t) \geq-q(s) f(x(\sigma(s))) \int_{s}^{t} \frac{z^{\prime}(\sigma(u)) \sigma^{\prime}(u)}{f(z(\sigma(u)))} \mathrm{d} u+L_{2} z(\sigma(t)) \frac{z^{\prime}(\sigma(s)) \sigma^{\prime}(s)}{f(z(\sigma(s)))}
$$




\section{BOUNDEDNESS AND OSCILLATION OF THIRD ORDER}

for $t \geq s \geq T$. Taking into account the monotonicity of $z, f, \sigma$, the last inequality may be rewritten to the form

$$
F_{s}^{\prime}(s, t) \geq-g(s) \frac{f(x(\sigma(s)))}{f(z(\sigma(s)))}[z(\sigma(t))-z(\sigma(s))]+L_{2} z(\sigma(t)) \frac{z^{\prime}(\sigma(s)) \sigma^{\prime}(s)}{f(z(\sigma(s)))}
$$

for $t \geq s \geq T$. In view of $x \in N^{+}$from (2) we have that $x(\sigma(s)) \geq z(\sigma(s))$ and then

$$
\frac{f(x(\sigma(s)))}{f(z(\sigma(s)))} \geq 1
$$

for all sufficiently large $s$. Therefore

$$
F_{s}^{\prime}(s, t) \geq-q(s)[z(\sigma(t))-z(\sigma(s))]+L_{2} z(\sigma(t)) \frac{z^{\prime}(\sigma(s)) \sigma^{\prime}(s)}{f(z(\sigma(s)))}
$$

for $t \geq s \geq T$. Combining (7), (8) and then integrating from $\sigma(t)$ to $t$ with respect to $s$ we obtain

$$
0 \geq L_{2} z(\sigma(t))\left[\int_{\sigma(t)}^{t} q(s) \int_{\sigma(s)}^{\sigma(t)} \frac{1}{r_{1}(u)} \int_{u}^{\sigma(t)} \frac{1}{r_{2}(v)} \mathrm{d} v \mathrm{~d} u \mathrm{~d} s+\int_{z(\sigma(\sigma(t)))}^{z(\sigma(t))} \frac{\mathrm{d} w}{f(w)}\right]
$$

for $t \geq s \geq T$. Because $\lim _{t \rightarrow \infty} z(t)=a>0$, we get

$$
\lim _{t \rightarrow \infty} \int_{z(\sigma(\sigma(t))}^{z(\sigma(t))} \frac{\mathrm{d} w}{f(w)}=0 .
$$

Then in view of the sign property of $L_{2} z(t)$ we obtain from (9) that

$$
\limsup _{t \rightarrow \infty} \int_{\sigma(t)}^{t} q(s) \int_{\sigma(s)}^{\sigma(t)} \frac{1}{r_{1}(u)} \int_{u}^{\sigma(t)} \frac{1}{r_{2}(v)} \mathrm{d} v \mathrm{~d} u \mathrm{~d} s \leq 0,
$$

which contradicts the condition $\left(\mathrm{H}_{6}\right)$. Therefore $\lim _{t \rightarrow \infty} z(t)=0$ and according to the Lemma 2.2 we have that $\lim _{t \rightarrow \infty} x(t)=0$.

Theorem 3.2. Let $\left(\mathrm{H}_{1}\right)-\left(\mathrm{H}_{5}\right)$ hold. Let there exist an integer $n \geq 0$ such that

$\left(\mathrm{H}_{7}\right) \quad \limsup _{t \rightarrow \infty} \int_{\sigma(t)}^{t} q(s) \int_{\sigma(s)}^{\sigma(t)} \frac{1}{r_{1}(u)} \int_{u}^{\sigma(t)} \frac{1}{r_{2}(v)} \mathrm{d} v \mathrm{~d} u \mathrm{~d} s>\frac{1-p}{1-p^{n+1}} \limsup _{u \rightarrow 0} \frac{u}{f(u)}$. 


\section{BOŽENA MIHALÍKOVÁ - EVA KOSTIKOVÁ}

Then every bounded solution $x$ of equation (1) is oscillatory.

Proof. Let $x$ be a bounded non-oscillatory solution of (1) on the interval $\left[t_{0}, \infty\right)$. Without loss of generality we may assume that $x(t)>0, x(\sigma(t))>0$ for $t \geq t_{1} \geq t_{0}$. We can proceed exactly as in the proof of the Theorem 3.1. In the case II. (b) we use the fact that $\left(\mathrm{H}_{7}\right)$ implies $\left(\mathrm{H}_{6}\right)$ and so we get a nonoscillatory solution with the properties $x(t)>0, z(t)=L_{0} z(t)>0, L_{1} z(t)<0$, $L_{2} z(t)>0, L_{3} z(t)<0, t \geq t_{2}\left(t_{2} \geq t_{1}\right.$ is sufficiently large) and $\lim _{t \rightarrow \infty} z(t)=$ $0, \lim _{t \rightarrow \infty} x(t)=0$. Using (2) we get

$$
x(t)=z(t)+p x(\tau(t))=z(t)+p z(\tau(t))+p^{2} x\left(\tau^{2}(t)\right)
$$

for all sufficiently large $t$. Repeating this procedure and using $\left(\mathrm{H}_{2}\right)$ and the monotonicity of $z$ we obtain that there exists an integer $n \geq 0$ such that $\tau^{n+1}(\sigma(t)) \geq t_{2}$ and

$$
x(\sigma(t))=\sum_{j=0}^{n} p^{j} z\left(\tau^{j}(\sigma(t))\right)+p^{n+1} x\left(\tau^{n+1}(\sigma(t))\right) \geq K z(\sigma(t)),
$$

where $K=\sum_{j=0}^{n} p^{j}>0, t \geq T \geq t_{2}$. Hence $f(x(\sigma(t))) \geq f(K z(\sigma(t)))$, integrating the equation (11) from $\sigma(t)$ to $t$ we have

$$
0<L_{2} z(t) \leq L_{2} z(\sigma(t))-M K \int_{\sigma(t)}^{t} q(s) z(\sigma(s)) \mathrm{d} s,
$$

where $t \geq T_{1} \geq T, M_{3}=\inf \left\{\frac{f(u)}{u} ; 0 \leq|z| \leq|K z(\sigma(T))|\right\}$. The inequality (7) yields

$$
-z(\sigma(s)) \leq-L_{2} z(\sigma(t)) \int_{\sigma(s)}^{\sigma(t)} \frac{1}{r_{1}(u)} \int_{u)}^{\sigma(t)} \frac{1}{r_{2}(v)} \mathrm{d} v \mathrm{~d} u
$$

where $t \geq s \geq T_{1}$. Combining (10) and (11) we obtain

$$
0<L_{2} z(\sigma(t))-L_{2} z(\sigma(t)) M K \int_{\sigma(t)}^{t} q(s) \int_{\sigma(s)}^{\sigma(t)} \frac{1}{r_{1}(u)} \int_{u}^{\sigma(t)} \frac{1}{r_{2}(v)} \mathrm{d} v \mathrm{~d} u \mathrm{~d} s,
$$

Because $L_{2} z(\sigma(t))>0$ we have

$$
1>M K \int_{\sigma(t)}^{t} q(s) \int_{\sigma(s)}^{\sigma(t)} \frac{1}{r_{1}(u)} \int_{u}^{\sigma(t)} \frac{1}{r_{2}(v)} \mathrm{d} v \mathrm{~d} u \mathrm{~d} s
$$

for $t \geq s \geq T_{1}$ and this contradicts the condition $\left(\mathrm{H}_{7}\right)$. 
Theorem 3.3. Let $\left(\mathrm{H}_{1}\right)-\left(\mathrm{H}_{6}\right)$ hold and in addition

$$
\limsup _{t \rightarrow \infty} \int_{\sigma(t)}^{t} q(s) \int_{\sigma(s)}^{\sigma(t)} \frac{1}{r_{1}(u)} \int_{u}^{\sigma(t)} \frac{1}{r_{2}(v)} \mathrm{d} v \mathrm{~d} u \mathrm{~d} s>(1-p) \limsup _{u \rightarrow 0} \frac{u}{f(u)} .
$$

Then the conclusion of the Theorem 3.2 holds.

Proof. Denote

$$
a=\limsup _{t \rightarrow \infty} \int_{\sigma(t)}^{t} q(s) \int_{\sigma(s)}^{\sigma(t)} \frac{1}{r_{1}(u)} \int_{u}^{\sigma(t)} \frac{1}{r_{2}(v)} \mathrm{d} v \mathrm{~d} u \mathrm{~d} s .
$$

Let the integer $n$ be chosen such that

$$
a>\frac{1-p}{1-p^{n+1}} \limsup _{u \rightarrow 0} \frac{u}{f(u)} .
$$

Then the assertion of this theorem follows from the Theorem 3.2 .

EXAMPLE 1. Let us consider the differential equation

$$
\begin{aligned}
& \left(\sqrt{t}\left(\sqrt{t}\left(x(t)-\frac{1}{2} x\left((\sqrt{t}-\pi)^{2}\right)\right)^{\prime}\right)^{\prime}\right)^{\prime} \\
& +\frac{3}{8 \sqrt{t}} x\left(\left(\sqrt{t}-\frac{3}{2} \pi\right)^{2}\right)=0, \quad t>\frac{27}{16} \pi^{2}
\end{aligned}
$$

All conditions of Theorem 3.3 are satisfied and all bounded solutions of the above equation are oscillatory. For instance, $x(t)=\sin \sqrt{t}$ is such solution.

\section{REFERENCES}

[1] BAČOVÁ, B.: On oscillation of third order linear neutral differential equations, Stud. Univ. Žilina, Math. Phys. Ser. 13 (2001), 7-15.

[2] CECCHI, M.-DOŠLÁ, Z.-MARINI, M.: Asymptotic behavior of solutions of third order delay differential equations, Arch. Math. (Brno) 33 (1997), 99-108.

[3] DOROCIAKOVÁ, B.: Some nonoscillatory properties of third order differential equations of neutral type, Tatra Mt. Math. Publ. 38 (2007), 71-76.

[4] DŽURINA, J.: Asymptotic properties of third order delay differential equations, Czech. Math. J. 45 (1995), 443-448.

[5] DŽURINA, J.-KOTOROVÁ, R.: Asymptotic properties of trinomial delay differential equations, Arch. Math. (Brno) 44 (2008), 149-158.

[6] ERBE, L. H.-KONG, Q.-ZHANG, B. G.: Oscillation Theory for Functional Differential Equations, Marcel Dekker, Inc., New York, 1994.

[7] JAROŠ, J.-KUSANO, T.: On a class of first order nonlinear functional differential equations of neutral type, Czech. Math. J. 40 (1990), 475-490. 


\section{BOŽENA MIHALÍKOVÁ - EVA KOSTIKOVÁ}

[8] MIHALÍKOVÁ, B.: A note on the asymptotic properties of systems of neutral differential equations, Stud. Univ. Žilina, Math. Phys. Ser. 13 (2001), 133-139.

[9] MOJSEJ, I.-OHRISKA, J.: On solutions of third order nonlinear differential equations, Cent. Eur. J. Math. 4 (2007), 154-163.

[10] RŮŽIČKOVÁ, M.- ŠPÁNIKOVÁ, E.: Oscillation theorem for neutral differential equations with quasi-derivatives, Arch. Math. (Brno) 30 (1994), 293-300.

Received October 7, 2008

Institute of Mathematics

Faculty of Science

P. J. Šafárik University

Jesenná 5

SK-041-54 Košice

SLOVAKIA

E-mail: bozena.mihalikova@upjs.sk chomka79@gmail.com 